\title{
FirstLight: Cosmological simulations of first galaxies at cosmic dawn
}

\author{
Daniel Ceverino ${ }^{1,2,3}$ (D) \\ ${ }^{1}$ Cosmic Dawn Center (DAWN) \\ ${ }^{2}$ Niels Bohr Institute, University of Copenhagen, Lyngbyvej 2, 2100 Copenhagen $\varnothing$, Denmark \\ ${ }^{3}$ Universitat Heidelberg, Zentrum für Astronomie, Institut fur Theoretische Astrophysik, \\ Albert-Ueberle-Str. 2, 69120 Heidelberg, Germany
}

\begin{abstract}
Cosmological hydrodynamical simulations have become an important theoretical tool for understanding the formation and evolution of the first galaxies during cosmic dawn, between redshifts 5 and 15. I will introduce the FirstLight database of about 300 zoom-in simulations with a resolution of 10 parsecs. This database agrees well with observed UV luminosity functions and stellar mass functions. I will discuss the origin and evolution of the star-forming main sequence of galaxies and the main drivers of the star formation histories at these early epochs. I will show simulated SEDs from UV to IR, including stellar and nebular emission. The restframe UV spectra show steep slopes and a high production efficiency of Lyman continuum photons. These properties are consistent with young stellar populations with low metallicities. Simulated recombination lines allow us to link the physical conditions of the gas around these stellar populations with observables, like equivalent widths in OIII or $\mathrm{H} \alpha$ or BPT diagrams at high-z. These simulations are making predictions that will be tested for the first time in future deep fields with the James Webb Space Telescope (JWST). I will finally discuss preliminary results involving JWST mock fields and predictions for ALMA observations by post-processing FirstLight snapshots with Powderday radiative transfer code.
\end{abstract}

Keywords. galaxies: evolution - galaxies: formation - galaxies: high-redshift.

\section{Introduction}

The period of cosmic dawn, the first billion years in the history of the Universe, is the final frontier for galaxy formation. Theory roughly predicts that the first galaxies form in the first gravitationally bound structures at high redshifts, $z \geqslant 12$. They quickly start to ionize their surroundings, driving the reionization of the Universe, which ends around $z \simeq 6$. However, little is known about these primeval galaxies. One of their most important properties is their spectral energy distribution (SED). In this talk, I will discuss SEDs coming from the FirstLight database of cosmological simulations of first galaxies at cosmic dawn, $z=5-15$ (Ceverino, Glover \& Klessen 2017). These cosmological simulations provide complex SFHs that are coupled with the non-uniform gas accretion history of galactic halos (Section 2 and Ceverino et al. 2018). The simulated SEDs are therefore consistent with the cosmological growth of structures at early times (Section 3 ). The final section discusses some results that can be found in Ceverino et al. (2018), such as BPT diagrams.

\section{Dwarfs at Cosmic Dawn $(z=6-15)$}

Galaxies at cosmic dawn look very compact (Fig.1). The stellar half-mass radius of this example is only $0.5 \mathrm{kpc}$. This is a factor 10 smaller than local galaxies with a similar mass. They are gas rich and they have typical $\mathrm{SFR} \simeq 20 M_{\odot} \mathrm{yr}^{-1}$, a factor 10 higher 


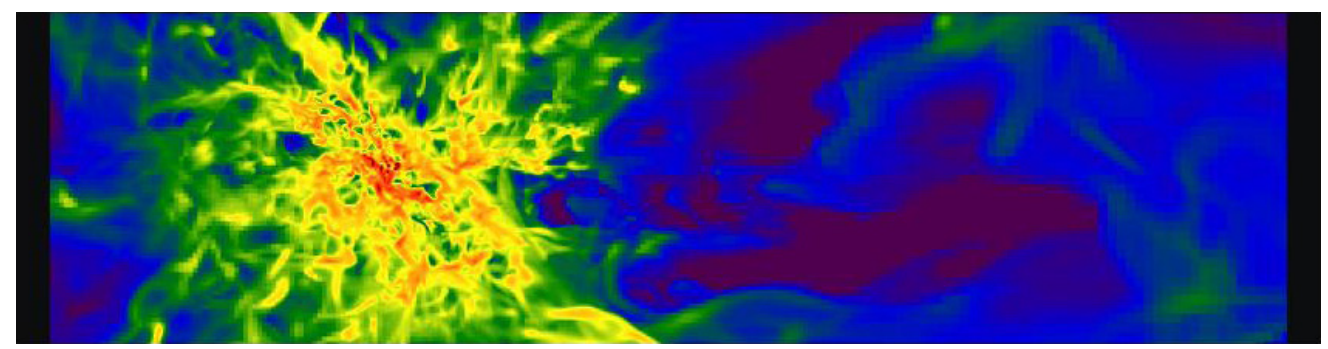

Figure 1. Gas distribution of a dwarf galaxy at $z=6$. The vertical size of the image is $3 \mathrm{kpc}$.

than their counterparts at $z=0$. This drives a clumpy and turbulent gas morphology, more similar to local starbursts. We have used the FirstLight database to study the Star Formation (SF) histories of $\sim 300$ galaxies with a stellar mass between $M_{*}=10^{6}$ and $3 \times 10^{9} M_{\odot}$ during cosmic dawn $(z=5-15)$.

The evolution of the SFR in each galaxy is complex and diverse, characterized by bursts of SF. Overall, first galaxies spend about $70 \%$ of their time undergoing SF bursts at $z>5$. This diversity sets the mean and scatter of the SFMS at $z \simeq 5-13$. High gas fractions and short gas depletion times are common during the SF bursts. The typical bursts at $z \simeq 6$ have a sSFR maximum of $5-15 \mathrm{Gyr}^{-1}$ with a duration of $\sim 100 \mathrm{Myr}$, one tenth of the age of the Universe. A quarter of the bursts populate a tail with very high sSFR maxima of $20-30 \mathrm{Gyr}^{-1}$ and significantly shorter time-scales of $\sim 40-80 \mathrm{Myr}$ at all masses. The mean period of time between consecutive bursts is $\sim 200 \mathrm{Myr}$ with a small mass dependence at $z \simeq 6$. The mean sSFR increases with redshift approximately as $s S F R \propto(1+z)^{5 / 2}$ at all masses, as predicted by $\Lambda$ CDM models. This is consistent with existing observations at $z \leqslant 8$. The typical sSFR height of a SF burst also increases with redshift, but it is always a factor $2 \pm 0.5$ higher than the mean sSFR at that redshift. This implies typical sSFR maxima of $s S F R_{\max }=20-30 \mathrm{Gyr}^{-1}$ at $z=9-10$. The tail of the distribution reaches $s S F R_{\max } \simeq 60 \mathrm{Gyr}^{-1}$ at these high redshifts. This evolution is driven by shorter time-scales at higher redshifts, proportional to the age of the Universe. These galaxies are the most efficient star formers in the history of the Universe.

\section{Example SEDs}

In this talk we focus on the intrinsic SEDs coming from the stellar populations and their surrounding, unresolved HII regions. They provide templates that could be used for a better understanding of the underlying stellar and gas properties in galaxies at cosmic dawn. Therefore, we ignore dust attenuation or any other radiative transfer effect from intervening gas. These effects will be considered in future works. We compute the SEDs of the simulated galaxies using publicly available tables from the Binary Population and Spectral Synthesis (BPASS) model (Eldrige et al. 2017) including nebular emission (Xiao et al. 2018).

Figure 2 shows two examples which represent the same galaxy with a stellar mass of $M_{*} \simeq 10^{8} M_{\odot}$ at $z \simeq 6$, at two different phases of evolution. The left panel depicts the galaxy at the peak of a burst of star formation. The starburst brings the galaxy a factor 6 times above the star forming main sequence at that redshift $\left(\mathrm{SSFR}=30 \mathrm{Gyr}^{-1}\right)$. Its luminosity at $1500 \AA$ is correspondingly high, $M_{1500} \simeq-19$. The nebular emission dominates the SED at all wavelengths. Lines, such as $\mathrm{Ly} \alpha, \mathrm{H} \alpha$, OIII or SiIII, are particularly prominent. For example, the equivalent width (EW) of $\mathrm{H} \alpha+\mathrm{NII}$ exceeds $1000 \AA$. OIII $+\mathrm{H} \beta$ is the next prominent feature with $\mathrm{EW} \simeq 700 \AA$. These lines contaminate the continuum in the $\mathrm{B}$ and $\mathrm{R}$ bands. The nebular continuum is also particularly important in the $\mathrm{U}$ band and it is able to completely remove the mass -sensitive Balmer break at $4000 \AA$ 

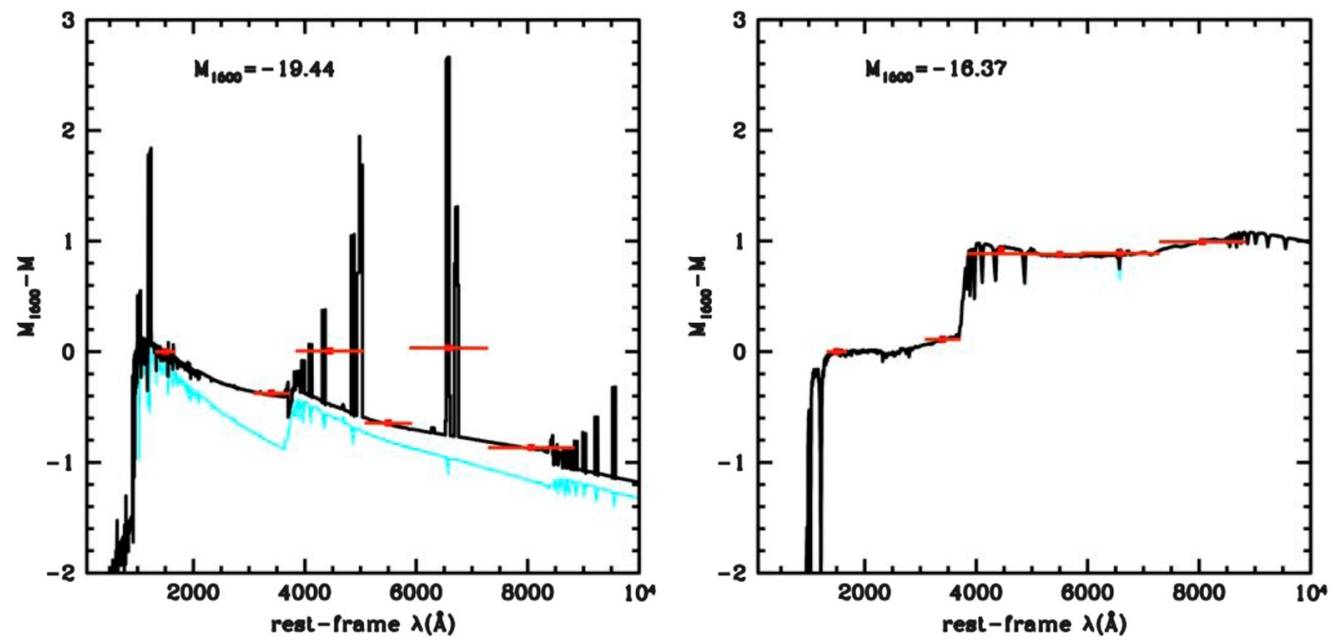

Figure 2. rest-frame SEDs of the same galaxy at the peak of a star formation burst at $z \simeq 6$ (left) and during the subsequent quiescent phase 200 Myr later (right). Cyan lines represent stellar light and black lines include nebular emission (lines+continuum). Red circles with bars represent six photometric bands. The monochromatic luminosity at each wavelength in $\mathrm{erg} / \mathrm{s} / \mathrm{Hz}$ is normalized to the absolute magnitude at $1500 \AA$ (top label).

The stellar continuum is also very steep, consistent with a young stellar population with extremely blue colors, $V-I=-0.2$ and $U-V=-0.4$. These are the typical features of an extreme emission-line galaxy at cosmic dawn. The SED of the same galaxy $200 \mathrm{Myr}$ after the starburst looks very different (right panel of Figure 2). The starburst and the subsequent feedback have quenched star formation significantly $\left(\mathrm{SSFR}=0.02 \mathrm{Gyr}^{-1}\right)$, placing the galaxy well below the star-forming main sequence. The SED confirms this quiescent nature. The luminosity at 1500 Ais very low, $M_{1500} \simeq-16$, for that stellar mass. There is no significant nebular emission. Instead, there is a strong Balmer break with significantly red rest-frame colors, $V-I=0.2$ and $U-V=0.8$, typical of a mature population.

\section{BPT Diagrams}

The nebular emission lines give some clues about the properties of the HII regions in galaxies using line luminosity ratios like $\operatorname{OIII}(5007 \stackrel{\circ}{\AA}) / \mathrm{H} \beta$ and $\mathrm{NII}(6584 \AA) / \mathrm{H} \alpha$. In Figure 3 we make predictions of BPT diagrams. The FirstLight galaxies form a clear sequence at $z=6$. The most massive galaxies, $M_{*} \simeq 1-2 \times 10^{9} \mathrm{M}_{\odot}$ occupy the tip of the sequence with $\mathrm{OIII} / \mathrm{H} \beta \simeq 0.5$ dex and $\mathrm{NII} / \mathrm{H} \alpha \simeq-1.5$ dex. These values are consistent with the line ratios of typical HII regions in low-metallicity dwarf galaxies at $z=0$ (van Zee \& Haynes 2006). These regions have relatively high luminosities in OIII with respect to $\mathrm{H} \beta$. Therefore, strong OIII-emitters at low- $z$ could be good analogs of galaxies responsible for reionization (Fletcher et al. 2018), although none of our galaxies reaches the highest values of $\mathrm{OIII} / \mathrm{H} \beta \simeq 1$ dex found in some of these analogs. This is mostly due to the low-metallicity of the primeval galaxies.

Low-mass galaxies have low luminosity ratios, reaching extremely low values for $M_{*} \simeq$ $10^{6} \mathrm{M}_{\odot}: \mathrm{OIII} / \mathrm{H} \beta \simeq-0.5$ dex and $\mathrm{NII} / \mathrm{H} \alpha \simeq-2.75$ dex. This is mostly driven by the low nebular metallicities of these low-mass galaxies (right panel of Figure 3), which reach $\log \left(\mathrm{Z}_{\mathrm{g}} / \mathrm{Z}_{\odot}\right)=-2$ for the smallest galaxies in the sample. More massive galaxies, $M_{*} \simeq 10^{8} \mathrm{M}_{\odot}$, still have lower ratios than $\mathrm{z}=0$ galaxies due to their low (10\% solar) metallicities. 

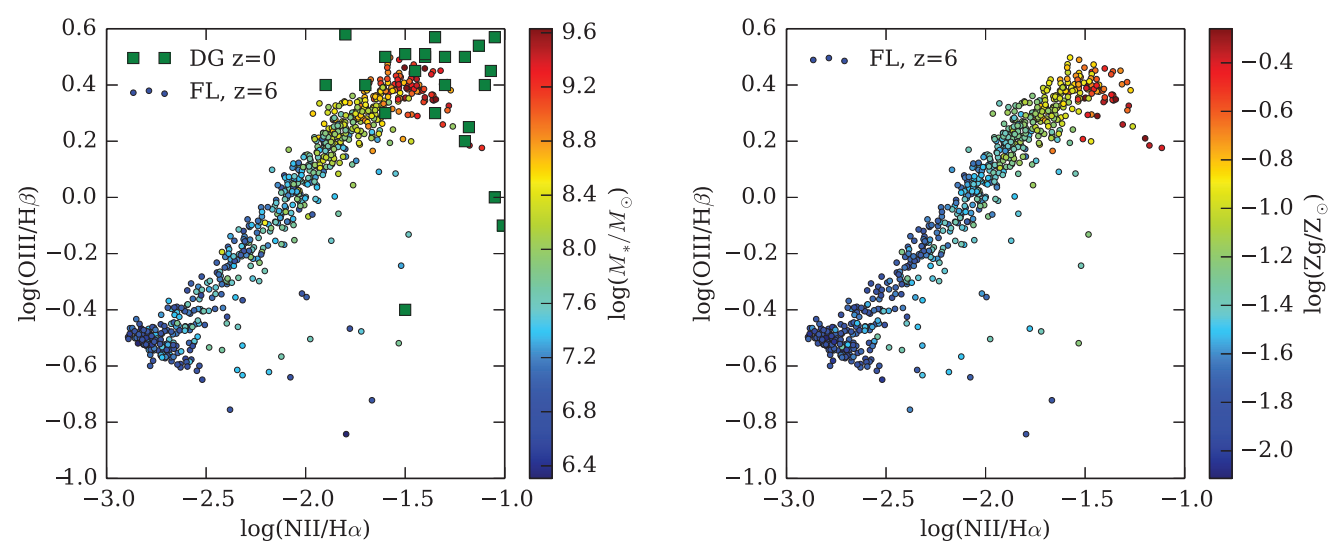

Figure 3. BPT diagrams at $z=6$ coloured by stellar mass (left), and mean gas metallicity (right). Massive galaxies are consistent with local dwarfs galaxies of similar mass (van Zee \& Haynes 2006). Low nebular metallicities drive the sequence towards very low metal-line luminosities.

\section{References}

Ceverino, D., Glover, S. C. O., \& Klessen, R. S. 2017, MNRAS, 470, 2791

Ceverino, D., Klessen, R. S., Glover, S. C. O., et al. 2018, MNRAS, 480, 4842

Ceverino, D., Klessen, R., \& Glover, S. 2019, MNRAS, 484, 1366

Eldridge, J. J., Stanway, E. R., Xiao, L., McClelland, L. A. S., Taylor, G., Ng, M., Greis, S. M. L., \& Bray, J. C., 2017, Publ. Astron. Soc. Australia, 34, e058

Fletcher, T. J., Robertson, B. E., Nakajima, K., Ellis, R. S., Stark, D. P., \& Inoue, A., 2018, preprint, p. arXiv:1806.01741

Xiao, L., Stanway, E. R., \& Eldridge, J. J. 2018, MNRAS, 477, 904

van Zee, L. \& Haynes, M. P. 2006, ApJ, 636, 214 